\title{
Physical Testing in an Elementary Engineering Camp
}

\section{Chelsea J. Andrews, Tufts University}

Chelsea Andrews is a graduate student at Tufts University pursuing a Ph.D. in STEM education. She received a B.S. from Texas A\&M University in ocean engineering and an S.M. from MIT in civil and environmental engineering. Her current research includes investigating how children naturally engage in engineering design through in-depth case study analysis. 


\section{Physical Testing in an Elementary Engineering Camp (Research to Practice) Strand: Principles of K-12 Engineering Education and Practice}

A common feature of many elementary classroom engineering design tasks is the use of a physical test to evaluate groups' design constructions. The design constructions often fail the physical test - they do not meet the design criteria; this is also very common in the professional world, at which point engineers diagnose the problem, then re-design and create an improved construction. Literature on elementary engineering has tended to contrast how students and professionals respond to failure, suggesting changes to the way engineering lessons are taught that will elicit responses from students more closely aligned with those of professionals $s^{1,2,3,4}$. What is missing in these studies is an in-depth look at what young students naturally do in response to the results of physical tests in engineering design situations. This paper analyzes a case study with an alternative focus, that of looking at what students are capable of and what resources they bring to engineering design tasks, centering the students and removing the comparison with adults.

This study presents an analysis of video data of a pair of fourth grade students working on a design task in an engineering summer camp. From a summary of their failure responses, they appear to be troubleshooting in an unfocused way and not learning from previous results indeed, far from what we would expect from professionals. However, if we instead analyze the single pair as an in-depth case study and look closely at their conversations in the context of the imposed material and temporal limits, it is possible to see how their responses to the failures proceed logically from their ideas about how the physical world works and what has occurred in the design process. This is consistent with the Piagetian position that within a localized context children behave logically ${ }^{\text {eg. }, 5,6}$. The analysis specifically focuses on the connections in each design cycle between (a) the actions and discussions of the group during planning and building, before their design construction fails the physical test, (b) their evaluation of their test results, and (c) their response to the failures of their design construction. This case study demonstrates the limitations of the prevailing reductionist approach to engineering education research and what can be gained from an in-depth situative analysis that looks closely at the students' discourse. Specifically, we concentrate on the resources students bring to dealing with the test and redesign cycle. Through transcript excerpts and pictures of their design constructions, we show that this pair of students is able to observe and evaluate the test results, discuss options for redesigning, appeal to the test result to make their cases, and complete the redesign before testing again. Beyond theoretical implications, these findings have important instructional implications for both engineering curriculum design and teaching practices.

\section{Background}

Engineering tasks are implemented with various objectives in mind, including improved performance in math or science and increasing the number and diversity of students interested in engineering ${ }^{7}$. Even so, classroom observations, descriptions from published research ${ }^{\text {e.g., } 2}$, as well as curricula descriptions (e.g., Engineering is Elementary ${ }^{8}$, Learning by Design ${ }^{9}$, and others ${ }^{7}$ ), have revealed that the overarching organization of classroom design tasks are generally quite similar. 
In these classrooms, a teacher presents the students with a fairly well-defined problem and tasks them with solving it by designing and creating a functional engineering solution. Very often, teachers also dictate the criteria and constraints of the problem and the physical tests that will be used to evaluate student solutions (although some classrooms are beginning to include students in this process ${ }^{\text {e.g., }}{ }^{10}$ ). Typically, pairs or teams of students then work together to plan and construct their designs from available materials. The groups of students then test their design constructions, and if time allows, iterate to improve their design. In this way, the classrooms loosely follow an engineering design process ${ }^{\text {e.g., } 11,12,13,14}$, although the steps are typically imposed by the teacher rather than arising organically from student needs. An example of a common engineering design task that typically follows this pattern is the egg-drop challenge, where students are tasked with creating an object out of craft materials that will allow an egg to survive a large fall.

There is often an assumption that when their constructions fail a physical test, students will engage in a simplified version of the steps professional engineers would take after a failure: to diagnose the problem with their design, decide how to address the problem, make the necessary changes, and then retest their modified design construction. However, this process may be difficult and unnatural for young and inexperienced designers. Crismond and Adams ${ }^{1}$ found that beginning designers evaluate their prototype's performances in an uncritical and unfocused way, which leads to ineffective troubleshooting and may result in over-valuing flawed prototypes. Sadler, Coyle, and Swartz ${ }^{2}$ found that fifth grade students benefit from tests with a "large dynamic range in measured performance"; otherwise, these students may misinterpret small changes that could be due to experimental error as legitimate feedback on their designs.

Sadler, Coyle, and Swartz ${ }^{2}$ did begin to look at the design trajectories of individual teams in terms of how they dealt with failure. In a design challenge to build the lightest effective paper truss, which essentially consists of systematically cutting out nonessential regions from a piece of paper, the authors noted that one team stopped trying after two failed attempts - the authors explain that the team members "were discouraged by their lack of success" (p. 306). There is an implication that the fault was in the team's strategy; specifically, in contrast to another team that failed in the first trial after beginning with a similarly "highly risky" approach but then backed off, the unsuccessful team "did not retreat from this failure and had another one" (p. 306).

Overall, the researchers had two main pieces of advice that seemed to be aimed at preventing this flawed strategy. The first is to conduct design challenges with many short iterations that provide small successes and failures, although they did not describe why this kept students engaged. The second point is adapted from Ford" ${ }^{15}$ : the class should have a "clear goal, universally understood and accepted" that will "inspire actions that students can evaluate from their own perspective" (Sadler, Coyle, and Swartz ${ }^{2}$, p. 313). Without a clear goal, students may, at the least, blindly follow teacher directions or, at worst, become disruptive and even break equipment.

These studies and others note observations about students' evaluation of the failures of their artifacts during physical tests that often occur as students engage in engineering design; however, they do not examine students' understandings of or reasoning about such failures. Our work is based on the need to conduct an in-depth examination of students' responses to failures during physical tests as they occur within a design process. The observations in these previous studies make it clear that instead of assuming that elementary students approach the designing and testing process with a simplified version of the professional design model, there is a need to 
investigate how students naturally go about these activities. In a complimentary approach, Crismond and Adams ${ }^{1}$ developed a "matrix of informed design" that compares the strategies of beginning and informed designers and suggests teaching strategies to guide beginners towards the informed strategies. While this comprehensive review characterizes the shortcomings of beginning engineers, it does not look at the resources beginning designers bring to the design process. When the emphasis is on how to turn students into experts, there is a focus on the students' deficits, what the students do differently that needs to be molded to be more like the experts. There is generally little discussion on whether what the fledgling designers are doing is logical considering the immediate situation they are in and how they believe the world works.

\section{Method}

One of the goals of this paper is to show, through an example, the benefits of using an in-depth case study approach for engineering education research. Employing a case study approach allows researchers to focus on and closely examine the discussions and actions of a single group over the course of an entire design task. Privileging depth (concentrating on a single or few cases) over breadth (looking at a larger number of cases) provides the space to deeply consider student thinking on a moment-by-moment basis ${ }^{16}$. We chose to focus on a single group of two students, Marco and Vincenzo, as they designed, built, tested, and iterated on their design construction during a single design task. Our approach involved transcribing the video data and analyzing the relationship between the evolution of their design construction and their interactions with each other, as well as with instructors and other students. To accomplish this, we drew on tools from discourse analysis ${ }^{17}$ to analyze the video data, including both verbal and non-verbal data as evidence. We were particularly vigilant about staying close to and trying to understand the students' experiences, rather than comparing their actions to those of experienced adult engineers. In this, our objective was to examine the logic — or lack thereof — of the students' design trajectory, in the localized context of the design task.

\section{Data source}

The data for this analysis comes from a week-long engineering design summer camp for students in grades 4-6 at the Center for Engineering Education and Outreach at Tufts University. This paper will focus on a single design task the students were given on the first day of the camp that lasted about 45 minutes. In this task, students worked in pairs or groups of three on their choice of one of two design challenges; the pair of students that is the focus of this paper, composed of two boys named Marco and Vincenzo, was the only group to choose the wind tunnel task. The goal of the wind tunnel task is to create an object out of craft materials that will hover in a plastic tube set above a vertically-oriented fan (see Figure 1) for about ten seconds without flying out the top or falling to the bottom. Because Marco and Vincenzo were the only group working on the wind tunnel task, they had nearly exclusive access to the testing station. 


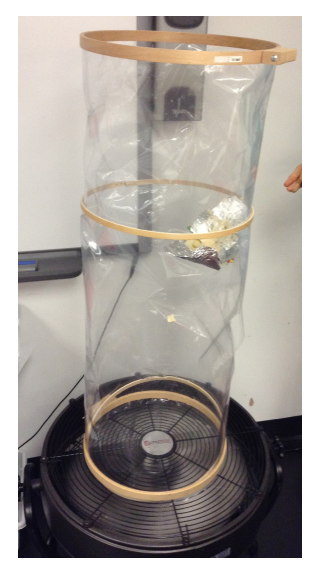

Figure 1: The test setup for the wind tunnel task

For this task, the students chose three craft materials they would use for their design construction before beginning to build. Marco and Vincenzo chose to use aluminum foil, wooden popsicle sticks, and masking tape (other options included straws, balloons, cardboard, and plastic wrap). They were allowed to use only these three materials, but they were permitted as much of these materials as they desired.

The two boys who are the focus of this study, Marco and Vincenzo, are first cousins who spend nearly as much time together as siblings. Their close relationship is one of the reasons they were selected for analysis, as even on the first day of camp, when many members of other groups were still shy with their partners, Marco and Vincenzo freely conversed and were comfortable enough with each other to disagree and debate design decisions. Also, because they were the only group to choose the wind tunnel task and thus had free access to the testing station, they were able to conduct more test-redesign cycles than many other groups.

\section{Findings}

In less than 30 minutes of build and testing time, the pair conducted seven tests of their design constructions, making changes or completely rebuilding after each of the seven tests. Their design construction failed the test every attempt until the final, public test, which is considered test eight. As the goal of the design task is for the design construction to hover within the plastic tube, a design construction can fail the test in two ways: by flying out of the top of the tube ("flies up") or by dropping to the bottom of the tube and landing on the fan cage ("falls").

For the first part of the analysis, we considered the summary of the pair's test results and responses, as provided in Table 1. For the seven tests the pair conducted while building, all of which were failures, twice their design construction failed by flying out of the top of the tube (test 1 and test 5) and for the other five tests the design construction fell to the bottom of the tube. Both times after their object flew out of the top, the pair altered the design construction by taping on additional popsicle sticks. In response to four of the five tests that resulted in the object falling to the bottom, the pair added foil onto their object. The remaining response (to test 3 ) was to scrap their current design construction and start building a new object from scratch. 
Table 1: Summary of test results and responses

\begin{tabular}{|c|c|c|c|}
\hline Test & Object & Result & Response to test \\
\hline 1 & & Flies up & Add 4 popsicle sticks (taped to foil) \\
\hline 2 & & Falls & Add extra set of foil wings \\
\hline 3 & & Falls & Start over \\
\hline 4 & & Falls & $\begin{array}{l}\text { Continue building; add foil wings (Note: } \\
\text { Just popsicle sticks taped together-didn't } \\
\text { really expect to work, see discussion } \\
\text { below) }\end{array}$ \\
\hline 5 & & Flies up & Add 6 popsicle sticks \\
\hline 6 & & Falls & $\begin{array}{l}\text { Add tin foil "patch" (mentioned starting } \\
\text { over again, but not enough time) }\end{array}$ \\
\hline 7 & & Falls & Add extra set of foil wings \\
\hline 8 & & Success & \\
\hline
\end{tabular}


When analyzed this way, it appears that Marco and Vincenzo have only two responses, one for each failure mode: they either add popsicle sticks when the object flies out the top or they add foil when the object falls. It would be simple to conclude that the pair is not learning from their past test results since they continue using the same responses as their constructions continue failing the test. They seem fixated on these two options and appear to be missing a whole range of other ideas; for example, they never change the size or shape of the design: both the original (up to test 3) and new design (after test 4) are composed of popsicle sticks in the center with foil "wings" extending to either side, and the overall dimensions of the object are very similar for every test. It would be legitimate to conclude that Marco and Vincenzo are falling into the trap of trying the same thing and expecting different results - certainly not what we would expect from experienced engineers.

Of course, idea fixation is only identified and criticized when the idea is not working. If the first solution you try for each problem works reasonably well, then it is not an issue to continue using that solution every time you encounter that problem. Even if there are other solutions that may be better options, if a certain solution is working, it is logical to keep using it, especially in a timelimited situation. If we look more closely at Marco and Vincenzo's tests and responses, we see that adding popsicle sticks after test 1 solved the problem they faced in that test, in the sense that in test 2 the object no longer flew out the top (although it still failed, they fixed the "fly-out-oftop' problem). Thus, in response to test 5 , when the object again flies out of the top, it is reasonable that they employ the same solution as after test 1: tape on additional popsicle sticks. While this line of reasoning explains the response to test 5 in terms of test 1 , it does not extend to the drop-to-bottom failures: adding an extra set of foil wings after test 2 did not fix the problem of the object falling, so it is counterintuitive that after test 6 and test 7 they again add foil.

By looking solely at the design trajectory summary, we can conclude that Marco and Vincenzo have only one response to each failure mode, and they seem fixated on a certain type of design, despite its consistent failure. In order to get a clearer picture of their design process, we will examine the discourse of Marco and Vincenzo as they test and alter their design construction. By looking at their conversations, we hope to uncover the reasons behind their design decisions and to shed light on some of the questions that arose in the more cursory initial analysis. For this case study approach, we will look at the response to each test in turn and provide pieces of transcript for analysis.

Test 1: In response to test 1, in which their design flew out of the top, Marco and Vincenzo immediately decide that it is too light and needs extra weight. While leaving the testing station, Marco declares "We have to put some more popsicle sticks and make it - add more weight" and Vincenzo agrees: "More weight? OK." They then debate where to add the extra popsicle sticks, and end up adding four in an " $\mathrm{X}$ " pattern on the foil wings of their design. Adding extra popsicle sticks for added weight is a reasonable response to the test, and because the design construction does not fly out of the top of the tube in the second test, we can conclude that this response was effective at solving that problem.

Test 2: In test 2, the altered design construction falls to the bottom of the tube. As he collects his construction from the bottom of the tube, Marco comments, "Too much weight," and when they return to their table Marco states his plan: "We have to make more wings, like, we're gonna make more tin foil wings. Quick quick make squares;" Vincenzo doesn't reply directly to these 
comments, and they begin looking for the materials to make more squares. In some respects, it seems counterintuitive that Marco and Vincenzo do not respond to this fall-to-bottom failure by removing some of the popsicle sticks they added before the test. This could be seen as evidence that the boys are approaching each failure as a unique event and not thinking in terms of their past design trajectory. Because they do not mention it in their speech, it is impossible to know whether they silently considered and rejected the idea of removing some popsicle sticks or whether that option was not thought of. Regardless, there is never one correct solution to a problem in these types of design tasks; it is more important to consider the actions they did take and the result. The pair added another layer of two wings above the original wings with a space between them (see Table 1 for a picture).

Test 3: After the design construction fails again by falling to the bottom in test 3, Marco and Vincenzo return to their seats and silently contemplate their object for a few seconds. Then Marco states, "OK, then I know exactly what to" and begins tearing off the foil, which surprises Vincenzo:

Vincenzo: What are you doing, dude?

Marco: Let's start over it's too big. Take off the materials and [unclear: possibly 'rip it']. The chopsticks are the only thing that [likely means popsicle sticks]

Vincenzo: OK, we need a new idea

Marco: You mean more like the hang glider idea?

Vincenzo: It's a good idea...you think we should change it or keep it?

Marco: Keep it. Like this time...I know what we're gonna do [12 sec pause] Should we do foil?

Vincenzo: Huh?

Marco: Should we do the foil one?...Nah. That one's gonna be even harder

Vincenzo: Yeah.

At first, it may seem strange to outside observers that Marco and Vincenzo are willing to tear apart their object and begin again after only three tests. From another perspective, it could be seen as remarkable that they started over at all, as other researchers have found that failure response to be rare: In a design task building bridges with fifth graders, $\operatorname{Roth}^{3}$ found that in over 50 projects, only one group chose to abandon their current design and begin again. This is consistent with the behavior of beginning designers described by Crismond and Adams ${ }^{1}$, some of whom "will not abandon their design ideas, even after running many tests and design iterations that clearly demonstrate a plan's ineffectiveness" (p. 767). They attributed this trend to ineffective diagnostic troubleshooting: because beginning designers do not actively look for "worrisome" patterns, they do not recognize "flawed performances," resulting in final designs that are "strikingly similar" to the initial plans (p. 767).

Instead of continuing on with their initial idea, Marco and Vincenzo are very willing to give up on a plan that does not seem to be working. When we also consider that the pair felt like they were under pressure due to the time constraint, as evidenced by their many references to not having enough time, it seems even more reasonable that they would not want to invest more time and energy into this design construction. Thus, regardless of whether or not from a professional engineering perspective this decision was optimal in this case, it is noteworthy that Marco and Vincenzo are willing to scrap a design and start again. 
After they decide to start again, which is unavoidable once Marco begins ripping the foil off, the pair discusses what they should do for their next plan. If we skip ahead to the object they end up with before test 5 , it is perhaps surprising that the new design construction is similar to the original design construction: both have popsicle sticks in the center with foil "wings" on either side. However, looking at the conversation above, we see in their dialogue that they do consider at least one other option, "the foil one" (although there is little explanation as to what that option entails), but that they decide that it would be "even harder." This dialogue shows that Marco and Vincenzo are perhaps thinking more divergently that it might appear from the design trajectory in Table 1.

Test 4: Test 4 is different than the other tests, as it appears that Marco and Vincenzo did not actually expect the design construction at this point to pass the test. The transcript shows that it was more of a test of opportunity. After Marco tapes together a row of popsicle sticks, he suddenly questions: "Wait, does this hover?," and gets up to go to the testing station. At the station before he tests, he tells Vincenzo, who has a doubtful expression on his face, "If this hovers I'll just be like" in an excited fashion. Both of these comments imply that he did not really expect the popsicle sticks to hover and it is unclear why it occurred to him to test it at all. Regardless, when they return to the table 20 seconds after they left to test, they simply continue on building, by agreeing to add wings:

Marco: $\quad$ Now we just put wings

Vincenzo: Yeah, now we just put wings

The way they continue working is further evidence that test 4 was more of an unexpected interruption to their building than a test of whether their design construction would work.

Test 5: After they add wings and test the new design construction, it flies out of the top like their first design did in test 1 . As they catch the design Vincenzo remarks: "Too light, too light" and Marco agrees: "Put more weight on it." They agree to add popsicle sticks, which was the same reaction they had after test 1 . As mentioned earlier, since that response worked after test 1 , in the sense that the object no longer had the problem of flying out of the top, it is reasonable that they employ that option again. However, they disagree on how many popsicle sticks are needed to fix their design. As Marco attempts to tape a pile of popsicle sticks to the middle of their design, Vincenzo tries to pull the tape away:

Vincenzo: Now don't put too many. No no no I don't think you should do that [trying to grab tape away]

Marco: We'll just put a mountain of tape. Put more right [pointing] stack stack. Dude we need a lot more tape. [Vincenzo is trying to cut tape, Marco moves his hand away] Didn't you see that thing fly?

The important thing about this exchange is how Marco appeals to the test as evidence backing up his stance that they need to add more popsicle sticks: "Didn't you see that thing fly?" While they never explicitly discuss what occurs during each test, all of their design changes are in direct reaction to the test results, and this comment reflects that shared understanding. After a bit more work, they encounter the same disagreement again, as Marco wants to tape on an additional "pack" of popsicle sticks: 
Marco: $\quad$ Tape that on

Vincenzo: Wait, dude, let's just test it with that first

Marco: Two packs

Vincenzo: No, dude, just test it

Marco: Yeah, we're going to test it

Vincenzo: Test it and THEN if it's too light we'll put this on

These exchanges and disagreements highlight the importance of the pair dynamic, which is often lost when comparing many groups across a classroom. When they test the new design (test 6), it falls to the bottom. If we only considered Marco and Vincenzo as a group, as when we are looking at the summary of the design trajectory, it appears that they simply added more popsicle sticks, just like they did after test 1 , and they failed again in test 6 , just like they did in test 2 . From this perspective, it looks as if they did not learn from their earlier experience. However, when we look at the exchanges above, we see that Vincenzo was in fact worried that they were adding too many popsicle sticks, and if Vincenzo had had more control over the situation, perhaps their design construction could have passed test 6 . It is only possible to explore this dynamic from looking closely at the group dialogue and not reducing the partners to a single entity.

Test 6: When they test the new design, with only one "pack" of popsicle sticks, the design construction falls to the bottom. Marco immediately makes a bid for more foil:

Marco: Dude, put more tin foil on.

Vincenzo: No

Marco: We're not wrecking this because we don't have time to make aVincenzo: - Well let's put a tin foil patch on the bottom

Marco: Yup. Patch tin foil on bottom. Interesting

While Marco, from the beginning, thinks that adding foil will help, Vincenzo is initially resistant. However, when Marco then says "We're not wrecking this because we don't have time to make a," and we can assume he was about to say something meaning "new one," Vincenzo then puts forth a new option: to "put a tin foil patch on the bottom," which Marco agrees to go along with.

One way to interpret this exchange is that Vincenzo initially does not want to put more tin foil on because he remembers that adding the foil wings after test 2 did not stop the object from falling in the next test. Vincenzo also may be beginning to doubt Marco's ideas, since it was Marco who wanted to add more wings after test 2 , who wanted to test just the popsicle sticks in test 4 , and who wanted to add even more popsicle sticks after test 5 . However, when Marco eliminates the other response they tried before - starting over-Vincenzo may then feel trapped by the time limit to find a relatively quick fix. It is not clear where the idea for the tin foil patch comes from, but it makes sense that Vincenzo does not want to add wings, because that did not work earlier, after test 2. We do not believe that Vincenzo is recalling the entire design trajectory in this level of detail, but we do think it is possible that Vincenzo recalls adding foil earlier, and it is quite likely that he remembers that every test thus far has failed, so it is reasonable to believe that he remembers adding foil did not succeed earlier. 
The next conversation (which occurs immediately after the above transcript), prompted by a teacher assisting with the camp, Maggie, helps explain their thoughts on why adding foil will help:

Maggie: What are you guys...what's your plan?

Marco: Well we just made it and now it's too heavy, so

Vincenzo: We're putting tin foil to lighten it

Maggie: And that is for this floating one over here? [pointing to wind tunnel test]

Vincenzo: Yeah

Maggie: So what do you mean you're putting tin foil to make it lighter?

Vincenzo: Because, um, the air pushes up on the tin foil and it's [unclear] makes it higher?

Maggie: Can you give me something in real life that's an example of that?

Marco: Uh, a hang glide - a glider

Vincenzo: A hang glider

Marco: A flying squirrel?

Maggie: Yeah, those are actually all really good examples, OK, perfect

Vincenzo's first explanation, "we're putting tin foil to lighten it" would point to a very simplistic conception, but his clarification "the air pushes up on the tin foil and it's [unclear] makes it higher" shows that he actually has a fairly developed understanding of what's happening; it's possible that he is beginning to understand how drag works. He seems to get that drag from the air is the force acting upwards on the object, and they certainly seem to understand that the downward force is the weight of the object. However, they seem to be missing the critical piece that to make the drag force stronger the air needs to act on a bigger area, which could be made of foil or any other material, as long as the total weight balances the drag force. So, unless air was flowing through the popsicle sticks beforehand (which seems unlikely) or air has a higher drag on foil than on popsicle sticks (not the case), adding the patch over the popsicle sticks would not help.

Almost a minute later they have taped on the foil patch and are ready to test it again:

Marco: Think it's good?

Vincenzo: Not yet, one more thing of tape

Marco: If it's too light we got the patch [holding the popsicle sticks he made before test 6]

Marco's comment here about the popsicle sticks he made before test 6 ("If it's too light we got the patch"), shows that Marco feels confident that the added tin foil patch will at least stop the object from falling to the bottom, and could even have too much of an effect, so that it may now be too light. However, when they test it (test 7) it falls again.

Test 7: As their design construction floats to the bottom, Vincenzo immediately states:

Vincenzo: Too heavy

Marco: $\quad$ More tin foil

Marco's immediate bid for more tin foil shows that, even though adding foil the last time did not work, Marco is still convinced that adding foil is the correct response when the object falls. The 
camp director then gave a one-minute warning a few seconds after they get back from the test. Marco is now taking charge of the building and mostly ignores Vincenzo. Marco flips the object over and adds another set of wings over the original set, on either side of the patch, and bends them so they are pointing up. They decide to test it, but as Marco gets up the camp director gives the announcement to stop building so they can do the final tests. Marco and Vincenzo then seem very upset and concerned that they will not be able to test their latest iteration:

Marco: [Quietly] What the hell

Vincenzo: I don't know, dude, I dunno

Marco: You think we lost?

Vincenzo: I dunno, dude, I dunno

Marco: Me neither

In this exchange Marco and Vincenzo seem very distressed and worried. After test 7, they had just failed twice in the same way (falling) and were running out of time. When Marco then adds wings, it could be that he is doing so as a last resort and because there is no time to think of something else, and not because he truly believes wings are the best option. Their nervousness about not being able to test their final design supports this conclusion. Thus, it is still possible that they remember that adding foil wings did not solve their design problem the first time they employed that response (after test 2), but given the context they are working in, their response seems reasonable.

\section{Discussion \& conclusion}

Much of the published engineering education literature summarizes results from the student teams; when this approach is used with this data, as seen in Table 1, it is very easy to conclude that this pair was suffering from idea fixation and was not learning from past test results. Based solely on the summary, this team appears to have a single response to each type of failure: adding popsicle sticks if the design construction flies out the top and adding foil if it falls to the bottom, plus starting over if the design fails in the same mode twice. Reusing the same responses after they have not worked in the past is certainly not what we would expect from experienced engineers or designers. From this analysis, it would be reasonable to conclude that these students need more guidance while designing and perhaps more structure to help them test and redesign more effectively.

However, when we take an in-depth look at what Marco and Vincenzo are actually discussing between tests the logic in their actions becomes apparent, especially when considering the full situation they are in, including time and material constraints. It is remarkable that they scrap their original design and start from scratch after test 3 , considering how rarely this has been seen by other researchers. After test 5, when the design construction fails by flying up, it is reasonable that they add more popsicle sticks, as that response was effective after they faced the same type of failure in test 1 . After test 6 , it at first seems strange that they are again adding more foil, but perhaps in their minds adding the patch is in a different category from adding wings, in which case they are trying a new option. Because they only have two materials to work with and adding wings was not effective the first time (after test 2), it is reasonable that they try another method of adding foil. After test 7, it again seems odd that they simply add wings, since that didn't work before, but when you consider their limited time frame and the fact that the patch didn't work, it 
makes sense that they would make something that they at least know how to create. The fact that they are nervous at the end may be a sign that they have not forgotten that wings did not work the first time (after test 2), and that they added them only as a last resort. Clearly, if they had full faith in the extra wings, they would not have whispered to each other, "What the hell" (quite strong language for these young boys) and, "You think we lost?" Even though they consistently met failure during the 30 minutes of building, they showed that they were able to productively engage in the test-redesign cycle: they could observe a failed test, discuss ways to re-design their construction in response to that test, make adjustments or completely recreate a new design, and finally test their new design construction.

Our goal is not to suggest that Marco and Vincenzo followed an "ideal" trajectory with this design task. Although it is possible to see the logic behind their choices, based on how they believe the world works and the options they felt they had available to them, they certainly did not explore the full design space of the task. They stuck to the basic idea of popsicle sticks in the middle with foil wings on the side throughout the entire task, even after they decided to start from scratch. They never mention the possibility of changing the overall size or shape of the object. If we were to compare their design trajectory to experienced engineers we would certainly find that Marco and Vincenzo come up far short; this comparison could lead to recommendations of scaffolding the design task in order to bring them closer to the expert model. However, we are arguing that comparing students to experienced or professional engineers or designers is less productive than viewing them as the young emerging designers they are. While their process may not reflect an expert engineer's, we believe that their resources for exploring, constructing, testing, and revising should be highlighted as valued abilities for navigating an engineering design process.

From an analysis of a case study of two fourth grade boys working on a design task in an engineering design summer camp, we have seen evidence that students are able to engage productively in the testing and redesign process with little adult intervention. Subject to the situation they were in, including limited time and material constraints, the students showed an ability to consider alternatives and debate these between themselves to come to compromises, appeal to the test results in making decisions, and recall past testing experiences when planning changes to their design construction. An important corollary to this claim, that these students were able to engage in the testing and redesign cycles constructively, is that this conclusion is difficult if not impossible to come by without using an in-depth case study approach.

1. Crismond, D., \& Adams, R. (2012). The informed design teaching and learning matrix. Journal of Engineering Education, 101(4), 738-797.

2. Sadler, P. M., Coyle, H. P., \& Schwartz, M. (2000). Engineering competitions in the middle school classroom: Key elements in developing effective design challenges. Journal of the Learning Sciences. 9(3), $299-327$.

3. Roth, W. -M. (1996). Art and artifact of children's designing: A situated cognition perspective. Journal of the Learning Sciences, 5(2), 129-166.

4. Welch, M. (1999). Analyzing the Tacit Strategies of Novice Designers. Research in Science \& Technological Education, 17(1), 19-34.

5. Schliemann, A. (1998). Logic of Meanings and Situated Cognition. Learning and instruction, 8(6), 549-560.

6. Karmiloff-Smith, A., \& Inhelder, B. (1975). If you want to get ahead, get a theory. Cognition, 3(3), $195-212$. 
7. Katehi, L., Pearson, G., \& Feder, M. (2009). Engineering in K-12 Education : Understanding the Status and Improving the Prospects.

8: Hester, K. and C. Cunningham (2007) Engineering is Elementary: An Engineering and Technology Curriculum for Children. American Society for Engineering Education Annual Conference and Exposition, Honolulu, Hawaii.

9: Kolodner, J.L., P. Camp, D. Crismond, B. Fasse, J. Gray, J. Holbrook, S. Puntambekar, and M. Ryan (2003). Problem-based Learning Meets Case-Based Reasoning in the Middle-School Science Classroom: Putting Learning by Design into Practice. The Journal of the Learning Sciences , 12(4), 495-547

10: McCormick, M. and Hynes, M. (2012) Engineering in a Fictional World: Early Findings from Integrating Engineering and Literacy. Conference Proceedings, Collection: Proceedings from the American Society of Engineering Education Annual Conference, San Antonio, Texas. Bibliography: Conference Proceedings

11. Ulrich, K. and S. Eppinger (2008) Product Design and Development, Fourth Edition, Boston: McGraw-Hill Higher Education

12. Dym, C., and P. Little (2004) Engineering Design: A Project-Based Introduction. Hoboken, New Jersey, John Wiley \& Sons, Inc.

13. Pahl, G. and Beitz, W. (1996) Engineering Design: A Systematic Approach, Second Edition, Springer, London

14. French, M.J. (1985) Conceptual Design for Engineers, The Design Council, London, and Springer-Verlag, Berlin

15. Ford, E. (1994). Discipline for home and school. Scottsdale, AZ: Brandt

16. Flyvbjerg, B. (2006). Five Misunderstandings About Case-Study Research. Qualitative Inquiry, 12(2), $219-245$. doi:10.1177/1077800405284363

17. Gee, J. P. (2005). An introduction to discourse analysis: Theory and method. (2nd edition) (pp. 1-19; 94-117). New York: Routledge. 